\title{
Personality, personnel selection and job performance
}

Dimitri van der Linden ${ }^{\mathrm{a}}$

Dirk Pelt ${ }^{\mathrm{a}}$, Curtis S. Dunkel ${ }^{\mathrm{b}}$, Marise Ph. Born ${ }^{\mathrm{a}}$

aDepartment of Psychology, Education, and Child Studies, Erasmus University Rotterdam, The Netherlands

bDepartment of Psychology, Western Illinois University, USA

\section{Synonyms}

Selection and assessment, work performance, personality traits

\section{Definitions}

Job Performance: The term job performance can either refer to the objective or subjective outcomes one achieves in a specific job (e.g., the profit of a sales persons, the number of publications of a scientist, the number of successful operations of a surgeon) or to workrelated activities (e.g., writing an article, conducting specific surgical acts). In the majority of research on this topic, job performance as an outcome is used.

Personnel selection: Personnel selection refers to the process of selecting the best employees for specific jobs.

\section{Main text}

One major application of personality research is in the area of personnel selection. The key question in this area is to which extent personality can predict how well a candidate will perform on the job he or she is applying for. Most scholars in this area acknowledge that personality has predictive validity for job performance. In line with this, personality assessment is part of the selection procedure in many organizations.

\section{The Big Five and job performance}

The majority of research on the role of personality in selection and assessment has been conducted using the well-known Big Five taxonomy, and there are now several meta-analyses showing that the Big Five show consistent, but low to moderate criterion-related validity regarding job performance (Barrick, Mount, \& Judge, 2001; Judge, Rodell, Klinger, Simon, \& Crawford, 2013). The most comprehensive study on this topic was conducted by Barrick, Mount, and Judge (2001). They conducted an extensive meta-analysis that ranged from $k=$ 143 studies $(N=23,225)$ for openness to experience, to $K=239(N=48,100)$ for 
conscientiousness. They included an overall (composite) score of job performance, but also many specific aspects of job performance (e.g., supervisor-rated and objective performance, and team performance) as well as a range of occupations (e.g., sales, police, management). Their findings clearly confirmed that conscientiousness is the broadest and most consistent job performance predictor of the Big Five dimensions (see Table 1). This result makes sense, as conscientiousness is characterized by traits such as dutifulness, persistence, and a tendency to work hard. Nevertheless, in absolute sense, the association between conscientious and overall job performance still remains moderate at best, with a meta-analytic and corrected (for unreliability, range restriction and sampling error) correlation of $\rho=.27$. The meta-analysis of Barrick et al. (2001) also showed that emotional stability (the reverse of neuroticism) was the only other Big Five dimension that displayed a significant (corrected) correlation with overall job performance. That is, its confidence interval did not include zero. For the other Big Five dimensions, namely extraversion, agreeableness and openness, the relationship with overall job performance was not significant as the confidence intervals all included zero. Several of the Big Five dimensions were significantly related to various types or aspects of job performance. For example, extraversion and openness were positively and significantly related to training performance, and agreeableness and extraversion both were positively and significantly related to team performance (see Table 1 for a full overview of all the findings).

After the Barrick et al. (2001) meta-analysis, several updates have been published. For example, Judge, Rodell, Klinger, Simon, and Crawford (2013) conducted a meta-analysis in which they included Big Five facets as well as the actual Big Five dimensions. However, none of the more recent meta-analyses made such an extensive distinction between job types and performance aspects as the original one by Barrick et al. (2001). Moreover, the criterionrelated validities that were reported in the more recent studies are rather similar to the ones reported in the 2001 meta-analyses. Therefore, it seems that the Barrick et al. (2001) study can, to date, still considered to be one of the major literature sources on the relationship between the Big Five and job performance.

One of the limitations of the Barrick et al. (2001) study was that they mainly reported associations with broad ratings or measures of performance and that they did not explicitly mention extra-role aspects of performance. Yet, the literature on this topic emphasizes that it would be useful to also consider work behaviors that fall outside the formal job description. This is exactly what was done in the meta-analyses of Berry, Ones, and Sackett (2007) and Chiaburu, Oh, Berry, Li, and Gardner (2011). The Berry et al. meta-analysis tested the associations between the Big Five and counterproductive work behavior (CWB) such as 
'cutting corners' or stealing from work. The Chiaburu et al. study examined the relationships between the Big Five and so-called organizational citizenship behavior (OCB), the tendency to take on additional duties, outside one's formal job description, in order to help colleagues or the organization. The results of these two meta-analyses are also summarized in Table 1 and show i) that all of the Big Five traits are positively related to most of the measures of $\mathrm{OCB}^{1}$ (with only few exceptions) and ii) that mainly conscientiousness, agreeableness, and emotional stability are negatively associated with CWB, whereas extraversion and openness are not clearly related to it.

Finally, Judge, Bono, Iles, and Gerhardt. (2002) and Bono and Judge (2004) conducted meta-analyses that looked at the associations between the Big Five and leadership behavior, which can also be considered a specific type of job performance (e.g. for managers). The results of these studies are summarized in Table 1 and show that four of the Big Five dimensions (agreeableness is the exception) demonstrated relevant positive relations to overall leadership ratings, and leadership emergence, whereas all Big Five dimensions were significantly related to leadership effectiveness and transformational leadership, the latter being a leadership style that many organizations consider to be an effective and desirable style.

All in all, the above described meta-analyses lead to the general conclusion that the Big Five, as one of the most established personality taxonomies can be used to predict a range of general and specific measures of job performance and may therefore also be relevant for selection procedures. On the other hand, effect sizes are often moderate at best, which is one of the reasons why some scholars hold the opinion that personality only has a limited use as a predictor of job performance (e.g. Morgeson, Campion, Dipboye, Hollenbeck, Murphy, \& Schmitt, 2007).

\section{Alternative personality taxonomies}

Although the Big Five is one of the leading personality models and is often assumed to provide a comprehensive description of personality, other traits outside of this model have been considered. Many of these 'alternative' traits have also been tested for their ability to predict job performance. It would go beyond the scope of the present section to describe all the findings on personality and job performance found outside the Big Five model. In contrast, a limited set of traits, that seem particularly relevant and promising in this area will

\footnotetext{
${ }^{1}$ OCB is positively related to emotional stability, but would show a negative association in case of neuroticism.
} 
be further discussed here, namely, the traits of the HEXACO model of personality, the dark triad, and emotional intelligence.

2.1 The HEXACO personality model. One model that seems to provide an increasingly accepted alternative for the Big Five model is the HEXACO model (Ashton \& Lee, 2007), which basically includes the Big Five, but has added an extra dimension to it, namely honesty-humility. As the label already indicates, this specific trait includes aspects such as the tendency to be honest and a lower drive for status and material gain. Many studies have now shown that this additional personality dimension can add to the prediction of job performance, particularly with regard to the ethical aspect of job performance (Johnson, Rowatt, \& Petrini, 2011; Lee, Ashton, \& De Vries, 2005). The honesty-humility dimensions seems to be a particularly good (negative) predictor of delinquent work tendencies and counterproductive work behavior. Basically, employees who score high on honesty-humility have a lower probability of engaging in socially undesirable behaviors at work such as betraying a colleague, stealing items from the workplace, or 'stretching the rules' in order to maximize the company's or their own benefits. As such, one can imagine that this personality dimension has add-on value in selection procedures, particularly when it involves jobs in which integrity constitutes a major component such as police officer, judge, or public sector manager.

2.2 Dark Triad. A more recent development in personality research is the explicit focus on dark side traits that are assumed to be associated with socially undesirable outcomes. In this line, there is an increasing stream on studies on the so-called Dark Triad (Paulhus \& William, 2002), which is a mix of the three intercorrelated negative traits; machiavelliansm, Narcissism, and psychopathy. Machiavelliansm refers to a trait characterized by an unconventional view on morality, low empathy, and the willingness to deceive and manipulate others for one's own gain. Narcissism relates to a tendency for self-enhancement or an unrealistic positive view on one's own personality or appearance. The trait Psychopathy consists of impulsiveness and thrill-seeking, combined with low empathy, anxiety, and a tendency towards being antagonistic. The three traits of the Dark Triad have in common low empathy, callousness, and a tendency to manipulate others. Due to this overlap, it currently remains an open debate whether it is more useful to consider the three traits individually or whether it would be better to focus on the shared variance of the traits (e.g. Glenn \& Sellbom, 2015), which is known to be of substantial size (i.e., somewhere between 50 to $75 \%$ shared variance). 
The nature of the relationship between the Dark triad and job performance is not straightforward and unidirectional, but may depend on the type of job and time frame (Spain, Harms, \& LeBreton, 2013). For example, due to their motivation and ability to manipulate others, individuals scoring high on one or more of the dark triad traits, may initially reach success as sales persons, managers, or in other jobs in which they need to influence others. Also, particularly psychoticism has been associated with high creativity and therefore may be positively associated with job performance or occupational success. For example, it is assumed that a substantial proportion of genius artists or scientists score relatively high on psychoticism. On the other hand, in the long term, employees scoring high on the dark triad may get into trouble due to their increased tendency towards delinquent behavior and problematic social relationships. There are many, sometimes very salient, examples of managers who were very high performers until it was discovered that they had acted illegally (e.g., stealing money) for reasons of self-enhancement or until they came into conflict with their subordinates and colleagues.

O’Boyle, Forsyth, Banks, and McDaniel (2012) conducted a large meta-analysis on the dark trait on the one hand, and job performance and counterproductive work behavior (CWB) on the other hand. They found that machiavelliansm and psychopathy both showed negative meta-analytic correlations with job performance, although the overall effect sizes were rather small ( $\rho=-.06$ and -.08 , respectively), possibly reflecting the complex link between the dark triad and performance as described above. Narcissism was not significantly associated with performance $(\rho=-.02)$. All three dark traits showed significant positive associations with CWB with effects sizes ranging from $\rho=.06$ (for P) to .35 (for $\mathrm{N}$ ).

Going beyond the direct link between the dark triad and job performance, it has been shown that individuals high on the dark triad tend to make career choices that optimize the utility of their traits and/or minimize negative consequences for themselves. For example, narcissists would be more likely to choose jobs that can lead to social approval and admiration, and machiavelliansts often avoid jobs that are unlikely to lead to high status (Jonason, Wee, Li, \& Jackson, 2014).

2.3 Emotional Intelligence. The last trait we discuss in this section is emotional intelligence (EI). EI started receiving increasing attention from scholars and practitioners in the nineties of the previous century. In its broadest definition, EI comprises either competencies and/or traits that are associated with adequately dealing with one own emotions and those of others. Initially, expectations of EI as a predictor of job performance were very high due to statements such as that for excellent job performance, EI may even be more 
important than cognitive abilities. However, those expectations were lowered in the following decades. One of the reasons for this was that it turned out to be rather difficult to obtain consensus on the theoretical definition of EI as well as it operationalization. Specifically, to date, some scholars argue that EI should mainly be considered as an ability and therefore should be classified and measured as such (Mayer, Caruso, \& Salovey, 2000). For example, one of the ruling definitions of EI as an ability states that it is “.... type of social intelligence that involves the ability to monitor one's own and others' emotions, to discriminate among them, and to use this information to guide one's thinking and actions” (Mayer, Salovey, \& Caruso, 2000, p. 139). Subsequently, it has been argued that EI can only reliably be measured with maximum performance tests, akin to how one would measure cognitive intelligence (i.e., by means of IQ tests).

Other researchers, however, consider EI as a trait and would argue that EI thus be classified under personality (Perze, Petrides, \& Furnham, 2005; Petrides \& Furnham, 2001). In this view, EI can be measured with surveys in a similar way as one would measure personality (by means of self-report). The current literature reveals no strong signs that a clear consensus on the definition and measurement of EI will be achieved soon. Several scholars have even expressed doubts about whether EI can be considered a distinctive trait or whether it merely is a configuration of existing traits such as personality and cognitive intelligence (e.g., Locke, 2005).

Despite these open questions on the nature of EI, in the current section it is relevant to discuss whether EI is related to job performance. One of the main sources of information on this topic is the meta-analysis of O’ Boyle, Humphrey, Pollack, Hawver, and Story (2011). One of the strengths of this study is that these authors included three major streams of EI measurement namely 1) an EI stream strictly based on ability definitions of EI and in which EI is only measured with maximum performance tests, 2) a stream in which EI is measured with self-report questionnaires, but based on the ability definition of EI, and 3) a stream based on trait definitions of EI and in which it is measured with questionnaires. They found that each of the three streams showed relevant validities for job performance with (meta-analytic corrected) hardly differing values of $\rho=.24$, 30, and .28, for streams 1 to 3 , respectively. The overall (averaged over the three streams) criterion-related validity of EI was .28.

Given the fact that some scholars would be skeptical about the notion that EI is anything else than combinations of traits such as the Big Five and cognitive ability, O’Boyle et al. (2011) also tested whether EI incrementally predicted job performance over and above those traits and abilities. They found that EI in the first stream (EI as ability) only contributed 
a non-significant $0.4 \%$ incremental validity beyond cognitive ability and the Big Five. EI measures in stream 2 and 3 did a better job, and showed significant incremental validities of $5.2 \%$ and $6.8 \%$, respectively. Based on the findings described above one may conclude that EI is a trait (or ability) which should be taken seriously with regard to predicting job performance.

\section{Determining the optimal level of trait measurement for predicting job performance:}

\section{The bandwidth fidelity discussion}

Irrespective of the specific personality model or traits one uses, a more general debate regarding the validity of personality relates to the so-called bandwidth-fidelity, which essentially addresses the question whether it is better to use narrow or broad traits to predict, in this case, job performance. More specifically, the basic dimensions in personality models always consist of presumed latent factors, extracted from lower-level measures of behavior. For example, one of the most widely used Big Five measures is the NEO-PI-R. The full version consists of around 200 items, which combine to up to 30 lower-level personality facets. Those facets, are then combined into five broader categories, the Big Five.

In many personality studies the default level that is taken into account is the Big Five (see previous sections). Nevertheless, some studies have suggested that when predicting job performance it may be better to use more narrow traits. This would be particularly relevant when one also uses relatively narrow criterion measures. In their seminal paper, Ones and Viswesvaran (1996) extensively addressed the bandwidth-fidelity debate and concluded that using relatively broad constructs would almost always be preferred above more specific, narrower, measures of personality. They provided several arguments for their conclusions. For example, because compared to broader measures, narrower measures, such as personality facets, often include less items they also show lower reliability. Combining facets into broader, latent constructs, however, increases reliability. Another argument was that scholars and practitioners tend to overestimate the unique features of different jobs. Ones and Viswesvaran (1996) argued that performance across various jobs is relatively strongly affected by general traits such as persistence, reliability, sociability, etc. Ones and Viswesvaran also argued that one often actually wants to predict a broad outcome such as general job performance. Such a broad criterion may best be predicted with broader constructs such as conscientiousness.

The view as expressed by Ones and Viswesvaran (1996) is, however, not shared by all scholars in this area. Several scholars uphold the view that personality facets may relevantly 
contribute to the prediction of performance beyond broader constructs. This would particularly be the case when predictor and criterion are aligned. That is, when one wants to predict a narrow aspect of performance (e.g., whether one is tidy at one's workplace) then it may be better to use a narrow trait (e.g., order). However, if one uses a broad, factorial complex criterion such as general job performance, then using a broader trait as predictor may lead to better validity. Given the findings on predictor and criterion alignment and the fact that some authors argue for the role of narrow traits, to date, the debate about the optimal level of measurement for the prediction of job performance remains active. Based on the their review of the bandwidth-fidelity debate in personnel selection, Rothstein and Goffin (2006) concluded that "...over the past decade a consensus is growing among researchers in this field that both broad and narrow personality measures may be effective predictors of job performance under the appropriate conditions.” (p. 163).

The main focus of the bandwidth-fidelity literature is on models such as the Big Five or the HEXACO and their underlying facets. A few studies, however, have also examined factors above the Big Five or HEXACO model. One of the first was of Ones, Schmidt, and Viswervaran (1994). They found that a composite measure of the dimensions conscientiousness, agreeableness, and emotional stability was a better predictor of job performance than any of the Big Five dimensions individually.

In the same line, the bandwidth-fidelity discussion has recently been expanded with the introduction of an even broader and relatively new concept in the field of personality, namely the general factor of personality (GFP). The notion of the GFP emerged from the observations that personality traits almost universally show relevant intercorrelations leading to a substantial component of shared variance (Figueredo et al., 2004; Rushton \& Irwing, 2012; Van der Linden, Te Nijenhuis, \& Bakker, 2010). In essence, the GFP captures the socially desirable ends of personality measures, which implies that, for instance in terms of the Big Five, high GFP-individuals, on average, could be characterized by being relatively sociable, industrious, friendly, emotionally stable, and open to new experiences.

Just as in the field of EI, research on the GFP currently has its share of debates about its nature. For example, several scholars consider the GFP to reflect a substantive factor that has strong relevance for understanding individual differences in personality. The currently leading substantive interpretation of this factor is in terms of general social effectiveness (e.g., Dunkel \& Van der Linden, 2013). The GFP would reflect knowledge about what is considered socially desirable or effective behavior and a tendency and/or ability to act in that way, thereby optimizing the obtainment of personal goals (e.g., getting a job or promotion, 
making friends, finding a desired partner). Other scholars, however, assume that the GFP merely arises due to methodological or measurement artifact and therefore does not have much relevance for understanding individual differences in behavior (e.g., Revelle \& Wilt, 2013).

Notwithstanding the various views on the nature of the GFP, the relevant question to address here is whether it relates to job performance and how it fits into the bandwidth fidelity debate. Regarding the former, there are several studies that have clearly shown that the GFP is associated with supervisor-rated and objective performance. The criterion-validity of the GFP on job performance seems rather favorable in comparison to, for example, the Big Five, with values that are often as high or higher than those of conscientiousness (Van der Linden et al., 2010). Recently, Pelt, Van der Linden, Dunkel, and Born (2016) reanalyzed the combined meta-analyses of Barrick et al. (2001) and Van der Linden et al. (2010) and, based on hundreds of studies, confirmed that the GFP has an overall corrected association with supervisor-rated and objective job performance of around $\rho=31$. The GFP also was associated with various aspects of performance and in various types of jobs (see Table 1).

Pelt et al. (2016) also tested the predictive value of the Big Five above and beyond the GFP and found that incremental validities of the Big Five were often low. For example, in a regression analysis using meta-analytic values, the GFP explained $10.2 \%$ of the variance in the overall job performance measure. Including the Big Five in the second step only led to an incremental validity of $2.4 \%$. In addition, each of the predictive validities of the individuals Big Five dimensions was strongly reduced after first including the GFP. For example, the initial meta-analytic correlation between emotional stability and overall job performance was $\rho=.15$ (see also paragraph 1 above), but was reduced to $\rho=-.01$ after controlling for the GFP. These findings seems to suggest that a large proportion of the predictive validity of specific personality traits may be due to their variance as captured by the GFP. When taking into account that the GFP may reflect general social effectiveness, these outcomes are not entirely surprising as it can be assumed that dealing with others and knowing how to act in order to convince other is a very important aspects of many jobs.

Sitser, Van der Linden, and Born (2014) currently is the only study that compared three different hierarchical levels of personality measures, including personality facets, the Big Five, and the GFP. Thus, this study included three 'bandwidth levels'. They concluded that when looking at overall performance of sales employees, the GFP was the best and most consistent predictor as it was related as strongly to supervisor rated as to objective (i.e. the number of new customers) sales performance. Yet, very specific aspects of the job could best 
be predicted with personality facets that were aligned with the criteria. Thus, the conclusion regarding the use of different personality levels remained similar to the one expressed by Rothstein and Goffin in 2006 (see above).

\section{Conclusion}

Based on the overview of the literature described above it can be concluded that personality can be used to predict job performance although the predictive values remain moderate to low. In response to this, there are ongoing attempts to improve the predictive validity of personality measures. One example is the use of a so-called frame of reference (FoR). FoR refers to a method in which one provides test-takers a clear context for filling in personality items. For example, most personality surveys assess how people tend to behave in general. However, in FoR-based research it is assumed that the behavior of individuals may differ across context. Someone may, for instance, act friendly and conscientious at work, but not at home. accordingly, it is hypothesized that one can increase the predictive validity of personality on job performance when one would explicitly ask in personality measures how one tends to behave at work. FoR interventions can occur to different levels. The simplest one would be to use a general personality instrument and explicitly instruct the test-takers to fill it in with their workplace behavior in mind. A more thorough FoR would be to add the context to each item. Thus instead of asking whether someone is generally sociable, one may explicitly ask whether someone is generally sociable at work. The most comprehensive FoR intervention is to completely reformulate personality items in such a way that they explicitly address work-related behavior (e.g., "I usually talk a lot with my colleagues”). The currently most comprehensive study on FoR effects is meta-analysis from Shaffer and Postlethwaite (2012). Taking into account 86 studies, they compared non-contextualized and contextualized personality measures with regard to their predictive validity on job performance. They reported that the average validity of non-contextualized personality measures was .11 (range .02 to .22), whereas the average validity of the contextualized measures was .24 (range .14 to .30). This would imply that using a FoR in personality questionnaires has a relevant and positive effect on the validity of personality measures.

An underutilized method for predicting job performance with personality is the use of other-ratings. Most studies on the predictive validity of personality on job performance have used self-report measures. However, this method has also yielded the extensive and complex discussion about faking and social desirability bias. Therefore, some scholars have emphasized the use of other-ratings of personality in this area. 
One of the most elaborate contemporary studies on this topic is from Connelly and Ones (2010), who meta-analytically compared self-reported and other-rated measures of personality. They concluded that the use of other-ratings of personality leads to substantial increase in the predictive validity of job performance. Moreover, other-rated personality measures showed significant incremental predictive validity above and beyond self-report measures of personality. Even though other-ratings would be useful, it is also clear that, in a selection context, this method includes several practical difficulties. Specifically, it is normally very difficult to obtain other-ratings of personality for candidates applying for a job.

\section{Cross references}

Organizational-Citizenship Behavior; Personality and counterproductive work behaviors; Personality and leadership; Personality and teamwork; Personality and work behavior; Teamwork

\section{References}

Ashton, M. C., \& Lee, K. (2007). Empirical, theoretical, and practical advantages of the HEXACO model of personality structure. Personality and Social Psychology Review, 11(2), 150-166.

Barrick, M. R., Mount, M. K., \& Judge, T. A. (2001). Personality and performance at the beginning of the new millennium: What do we know and where do we go next?. International Journal of Selection and assessment, 9(1-2), 9-30.

Berry, C. M., Ones, D. S., Sackett, P. R. (2007). Interpersonal deviance, organizational deviance, and their common correlates: A review and meta-analysis. Journal of Applied Psychology, 92, 410-424.

Bono, J. E., \& Judge, T. A. (2004). Personality and transformational and transactional leadership: A meta-analysis. Journal of Applied Psychology, 89, 901-910.

Chiaburu, D. S., Oh, I.-S., Berry, C. M., Li, N., \& Gardner, R. G. (2011). The five-factor model of personality traits and organizational citizenship behaviors: A meta-analysis. Journal of Applied Psychology, 96, 1140 -1166.

Connelly, B. S., \& Ones, D. S. (2010). Another perspective on personality: meta-analytic integration of observers' accuracy and predictive validity. Psychological Bulletin, 136, 1092-1122.

Dunkel, C. S., \& Van der Linden, D. (2014). Evidence for the general factor of personality as social effectiveness. Personality and Individual Differences, 64, 147-151. 
Figueredo, A. J., Vásquez, G., Brumbach, B. H., \& Schneider, S. M. R. (2004). The heritability of life history strategy: The K-factor, covitality, and personality. Social Biology, 51, 121-143.

Glenn, A. L., \& Sellbom, M. (2015). Theoretical and empirical concerns regarding the dark triad as a construct. Journal of personality disorders, 29(3), 360-377.

Johnson, M. K., Rowatt, W. C., \& Petrini, L. (2011). A new trait on the market: Honesty-Humility as a unique predictor of job performance ratings.Personality and Individual Differences, 50(6), 857-862.

Jonason, P. K., Wee, S., Li, N. P., \& Jackson, C. (2014). Occupational niches and the Dark Triad traits. Personality and Individual Differences, 69, 119-123.

Judge, T. A., Bono, J. E., Ilies, R., \& Gerhardt, M. (2002). Personality and leadership: A qualitative and quantitative review. Journal of Applied Psychology, 87, 765-780.

Judge, T. A., Rodell, J. B., Klinger, R. L., Simon, L. S., \& Crawford, E. R. (2013).

Hierarchical representations of the five-factor model of personality in predicting job performance: Integrating three organizing frameworks with two theoretical perspectives. Journal of Applied Psychology, 98, 875.

Lee, K., Ashton, M. C., \& de Vries, R. E. (2005). Predicting workplace delinquency and integrity with the HEXACO and five-factor models of personality structure. Human performance, 18, 179-197.

Morgeson, F. P., Campion, M. A., Dipboye, R. L., Hollenbeck, J. R., Murphy, K., \& Schmitt, N. (2007). Reconsidering the use of personality tests in personnel selection contexts. Personnel psychology,60, 683-729.

Locke, E. A. (2005). Why emotional intelligence is an invalid concept. Journal of Organizational Behavior,26, 425-431.

Mayer, J. D., Caruso, D. R., \& Salovey, P. (2000). Selecting a measure of emotional intelligence: The case for ability scales.

Murphy, K. R. (2005). Why don't measures of broad dimensions of personality perform better as predictors of job performance?. Human Performance,18, 343-357.

O'Boyle, E. H., Humphrey, R. H., Pollack, J. M., Hawver, T. H., \& Story, P. A. (2011). The relation between emotional intelligence and job performance: A meta-analysis. Journal of Organizational Behavior,32, 788-818.

O'Boyle Jr, E. H., Forsyth, D. R., Banks, G. C., \& McDaniel, M. A. (2012). A meta-analysis of the dark triad and work behavior: A social exchange perspective. Journal of Applied Psychology, 97, 557.

Ones, D. S., Schmidt, F. L., \& Viswesvaran, C. (1994). Do broader personality variables predict job performance with higher validity. In Conference of the Society for Industrial and Organizational Psychology, Nashville, TN, USA.

Ones, D. S., \& Viswesvaran, C. (1996). Bandwidth-fidelity dilemma in personality 
measurement for personnel selection. Journal of Organizational Behavior, 17, 609-626.

Paulhus, D. L., \& Williams, K. M. (2002). The dark triad of personality: Narcissism, Machiavellianism, and psychopathy. Journal of research in personality,36, 556-563.

Pelt, D. H. M., Van der Linden, D., Dunkel, C. S., \& Born, M. Ph. (2016). The general factor of personality and job performance: Meta-analytic evidence. Submitted.

Pérez, J. C., Petrides, K. V., \& Furnham, A. (2005). Measuring trait emotional intelligence. Emotional intelligence: An international handbook, 181-201.

Petrides, K. V., \& Furnham, A. (2001). Trait emotional intelligence: Psychometric investigation with reference to established trait taxonomies.European journal of personality, 15, 425-448.

Rothstein, M. G., \& Goffin, R. D. (2006). The use of personality measures in personnel selection: What does current research support?. Human Resource Management Review, 16(2), 155-180.

Spain, S. M., Harms, P., \& LeBreton, J. M. (2014). The dark side of personality at work. Journal of Organizational Behavior, 35, S41-S60.

Sitser, T., van der Linden, D., \& Born, M. P. (2013). Predicting sales performance criteria with personality measures: The use of the general factor of personality, the big five and narrow traits. Human Performance, 26, 126-149.

Van der Linden, D., te Nijenhuis, J., \& Bakker, A. B. (2010a). The general factor of personality: A metaanalysis of Big Five intercorrelations and a criterion-related validity study. Journal of Research in Personality, 44, 315-327. 
Table 1. Big Five Correlations With Criteria and Social Desirability Measures

Corrected correlations

Uncorrected correlations

\begin{tabular}{|c|c|c|c|c|c|c|c|c|c|c|c|c|}
\hline Variable & $\mathrm{O}$ & $\mathrm{C}$ & E & $\mathrm{A}$ & ES & GFP & $\mathrm{O}$ & $\mathrm{C}$ & $\mathrm{E}$ & A & ES & GFP \\
\hline \multicolumn{13}{|l|}{ Performance criteria $^{a}$} \\
\hline Overall job performance & .07 & .27 & .15 & .13 & .13 & .31 & .03 & .12 & .06 & .06 & .06 & .16 \\
\hline Supervisor rated performance & .07 & .31 & .13 & .13 & .13 & .33 & .03 & .15 & .07 & .06 & .07 & .19 \\
\hline Objective performance & .03 & .23 & .13 & .17 & .10 & .28 & .02 & .10 & .06 & .07 & .05 & .14 \\
\hline Training performance & .33 & .27 & .28 & .14 & .09 & .44 & .14 & .13 & .13 & .07 & .05 & .28 \\
\hline Team performance & .16 & .27 & .16 & .34 & .22 & .47 & .08 & .15 & .08 & .17 & .13 & .23 \\
\hline \multicolumn{13}{|l|}{ Contextual performance ${ }^{b}$} \\
\hline OCB - Composite & .17 & .22 & .11 & .17 & .15 & .30 & .11 & .14 & .07 & .11 & .10 & .23 \\
\hline OCB - Organizational & .19 & .20 & .02 & .19 & .12 & .26 & .13 & .13 & .01 & .12 & .08 & .20 \\
\hline OCB - Individual & .20 & .25 & .11 & .20 & .17 & .34 & .13 & .16 & .07 & .13 & .11 & .26 \\
\hline OCB - Change & .17 & .12 & .15 & -.03 & .09 & .18 & .11 & .08 & .10 & -.02 & .06 & .14 \\
\hline CWB - Composite & -.08 & -.35 & -.03 & -.44 & -.26 & -.47 & -.06 & -.30 & -.03 & -.35 & -.22 & -.46 \\
\hline CWB - individual deviance & -.09 & -.23 & .02 & -.46 & -.24 & -.40 & -.07 & -.19 & .02 & -.36 & -.20 & -.37 \\
\hline CWB - organizational deviance & -.04 & -.42 & -.09 & -.32 & -.23 & -.48 & -.03 & -.34 & -.07 & -.25 & -.19 & -.44 \\
\hline \multicolumn{13}{|l|}{ Leadership $^{c}$} \\
\hline Leadership & .24 & .28 & .31 & .08 & .24 & .46 & .16 & .20 & .22 & .06 & .17 & .31 \\
\hline Leader emergence $^{\dagger}$ & .24 & .33 & .33 & .05 & .24 & .49 & - & - & - & - & - & - \\
\hline Leadership effectiveness $^{\dagger}$ & .24 & .16 & .24 & .21 & .22 & .40 & - & - & - & - & - & - \\
\hline Transformational leadership & .15 & .13 & .24 & .14 & .17 & .32 & .11 & .10 & .19 & .10 & .15 & .30 \\
\hline \multicolumn{13}{|l|}{ Specific job types $^{a}$} \\
\hline Sales & -.03 & .25 & .11 & .01 & .05 & .20 & -.01 & .11 & .07 & .01 & .03 & .12 \\
\hline Management & .10 & .25 & .21 & .10 & .09 & 31 & .05 & .12 & .10 & .04 & .05 & .17 \\
\hline Professional & -.11 & .24 & -.11 & .06 & .06 & .29 & -.05 & .11 & -.05 & .03 & .04 & .17 \\
\hline Police & .03 & .26 & .12 & .13 & .12 & 13 & .02 & .13 & .06 & .06 & .07 & .07 \\
\hline Skilled/semi-skilled & .05 & .23 & .06 & .10 & .15 & .25 & .03 & .12 & .03 & .05 & .08 & .15 \\
\hline
\end{tabular}


${ }^{\text {a }}$ From Barrick et al. (2001).

${ }^{\mathrm{b}}$ OCB criteria from Chiaburu et al. (2011), CWB criteria from Berry, Ones and Sackett (2007).

${ }^{c}$ Leadership, leader emergence and leadership effectiveness from Judge et al. (2002), transformational leadership from Bono and Judge (2004).

${ }^{\dagger}$ No uncorrected correlations were provided in Judge et al. (2002).

$\mathrm{O}=$ openness to experience, $\mathrm{C}=$ conscientiousness, $\mathrm{E}=$ extraversion, $\mathrm{A}=$ agreeableness, $\mathrm{ES}=$ emotional stability, GFP = general factor of personality 